\title{
THE ADULT ANATOMY OF THE LYMPHATIC SYSTEM IN THE COMMON RAT (EPIMYS NORVEGICUS) ${ }^{1}$
}

\author{
THESLE T. JOB
}

From the Laboratories of Animal Biology, State University of Iowa

FOUR FIGURES

In the fall of 1914 the writer undertook the study of the origin and development of the lymphatic system in the common rat. Before the work had proceeded very far it was evident that a knowledge of the adult anatomy would be of no small amount of help in guiding and interpreting the work on the origin and development of this system. So the plan was changed to a study of the adult anatomy of the lymphatic system. This paper gives the chief results of the work.

Fifty rats have been examined in the course of the study. The lymphatic system was injected from the soles of the feet, the tip of the tongue, the lips, the walls of the intestines, the spleen, the lumbar, thoracic, inguinal, axial, intestinal and cervical lymph nodes. The first nineteen specimens were injected with India ink, using the hypodermic syringe for pressure. In the next six specimens, a solution of soluble blue was used, with a glass cannula and pressure bulb apparatus. The remainder of the work was done with Berlin blue gelatin mass, a small crystal each of thymol and potassium iodide being added to preserve and lower the melting point of the mass. The supply of gelatin mass was kept in a warm water bath, and the cannula occasionally warmed. Injections could be made through a much smaller aperture and in a much more satisfactory way by this method.

${ }^{1}$ An abstract of a thesis presented to the Graduate Faculty of the State University of Iowa for the degree of Master of Science. 


\section{RESULTS}

Injections made through the soles of the feet, showed a variable number of superficial lymph vessels joining to form larger lymph vessels which followed the main course of the radial and ulnar veins, or dorsal and plantar branches of the saphenous vein, to the elbow or knee lymph node $(1,2,3,4$, fig. 1). From the single node, 3 and 4 , the femoral lymph vessel continued to the lumbar node $(5-6)$. A branch is given off from this vessel at the juncture of the superficial epigastric vein and the femoral vein, which leads to the inguinal nodes $(7-8)$.

Just below the posterior end of the rectum is a small single node (30) which receives the lymph from the caudal region and appendages. From this node a vessel leads to the left lumbar node $(6)$, or to the left femoral lymph vessel, joining it at about the juncture of the iliac and inferior vena cava.

The lumbar nodes (5-6), which are normally double, lie just caudad of the ilio-lumbar veins, on either side of the vena cava. There is a great range of variation, however, in the position of these nodes, due, in the main, to the variability of the ilio-lumbar veins. If the veins are well caudad, the lymph nodes may be crowded back opposite to each other; or, if the veins are well

FIGURE 1

$1-2$, right and left elbow nodes

$3-4$, right and left knee nodes

$5-6$, lumbar nodes

$\gamma-8$, inguinal nodes

$9-10$, renal nodes

11 , cisterna chyli

12, cisterna group of lymph nodes

13 , intestinal node

14, thoracic duct

15 to 20 , axial nodes

23-24, jugulo-subclavian taps

25 , thoracic group

26, posterior cervical nodes

27 , anterior cervical nodes

28 , submaxillary nodes

29 , tongue and lip plexus

30 , caudal lymph node
FIGURES 2, 3

$a$, renal lymph vessel

$c i$, cisterna chyli; 12 , cisterna group

13 , intestinal node; $5-6$, lumbar nodes

$9-10$, renal nodes

p.c.v., posterior vena cava

$i-l$., ilio-lumbar vein

FIGURE 4

1, spleen; 2 , fundus lymph vessel

3 , pyloric lymph vessel

4 , intestinal lymph vessel

5 , connection with portal vein

6 , intestinal branch to cisterna chyli

7 , appendix; 8 , mesenteric nodes

9 , intestinal vessel

10 , mesenteric branches of lymph vessel

13 , intestinal node 


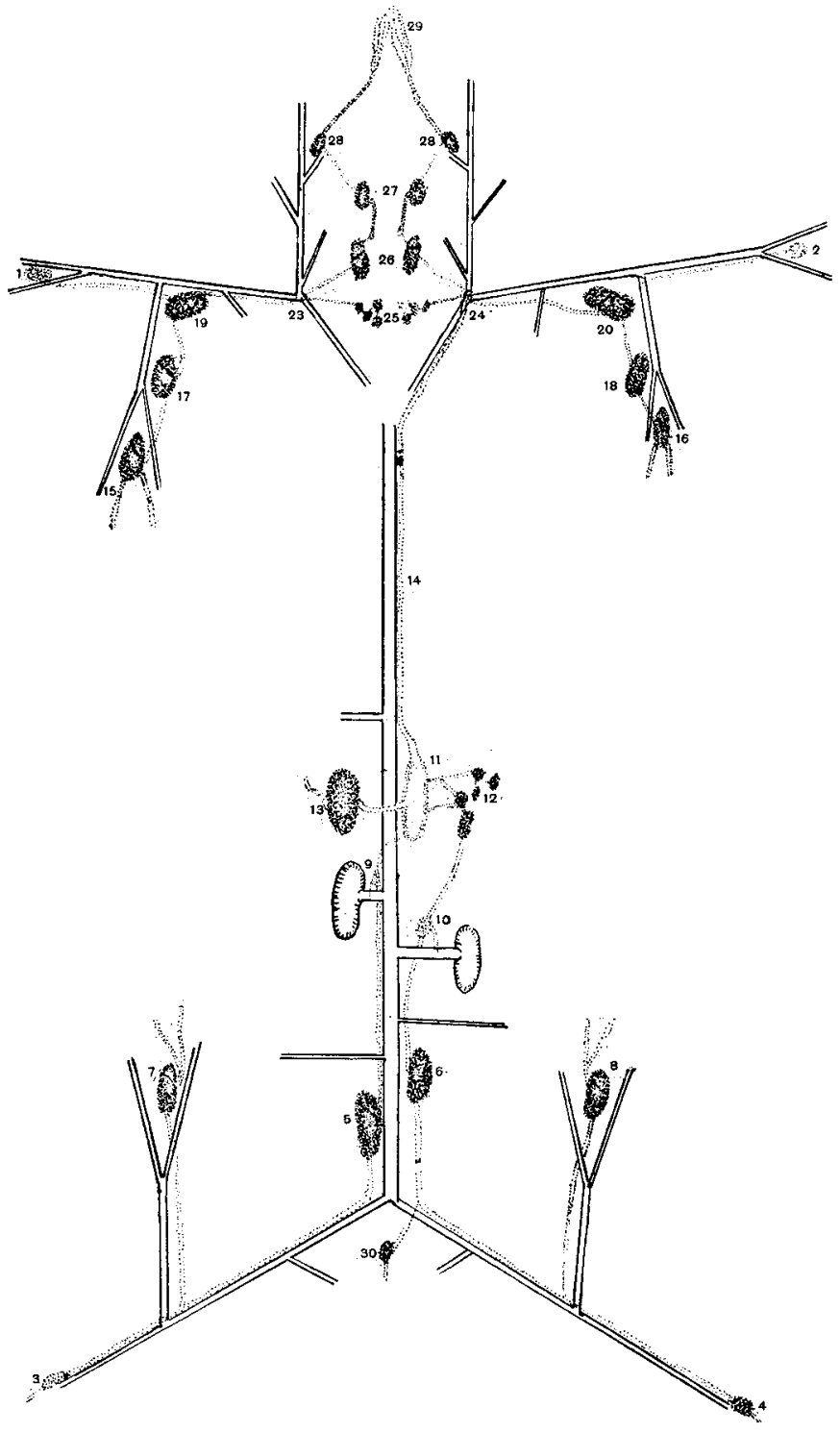

Fig. 1 Venous system shown diagrammatically in solid lines, the lymphatic system stippled. The lateral veins and lymph vessels are continuous, although not shown so in the figure. Lymph node 18 , the intestinal node, so marked in all drawings. 


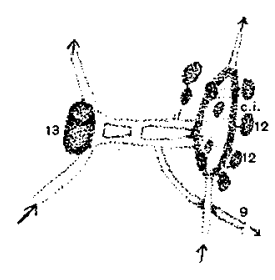

A
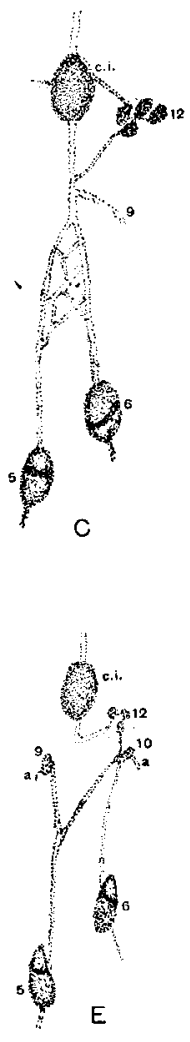
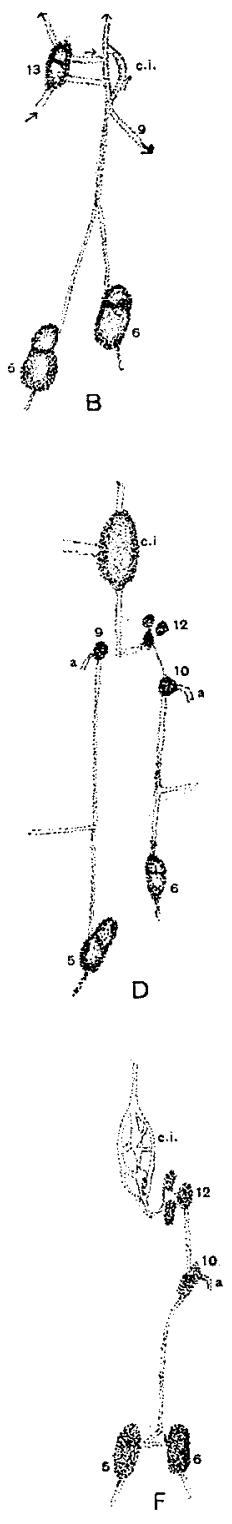


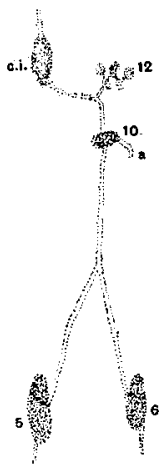

A

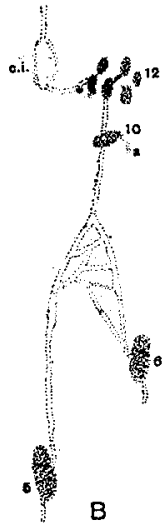

3

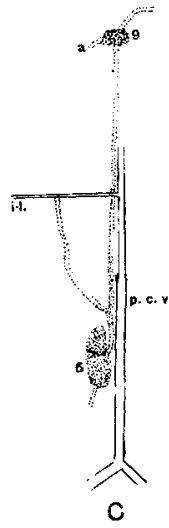

C

Figs. 2, 3 Exceptional and type variations of the lumbar region

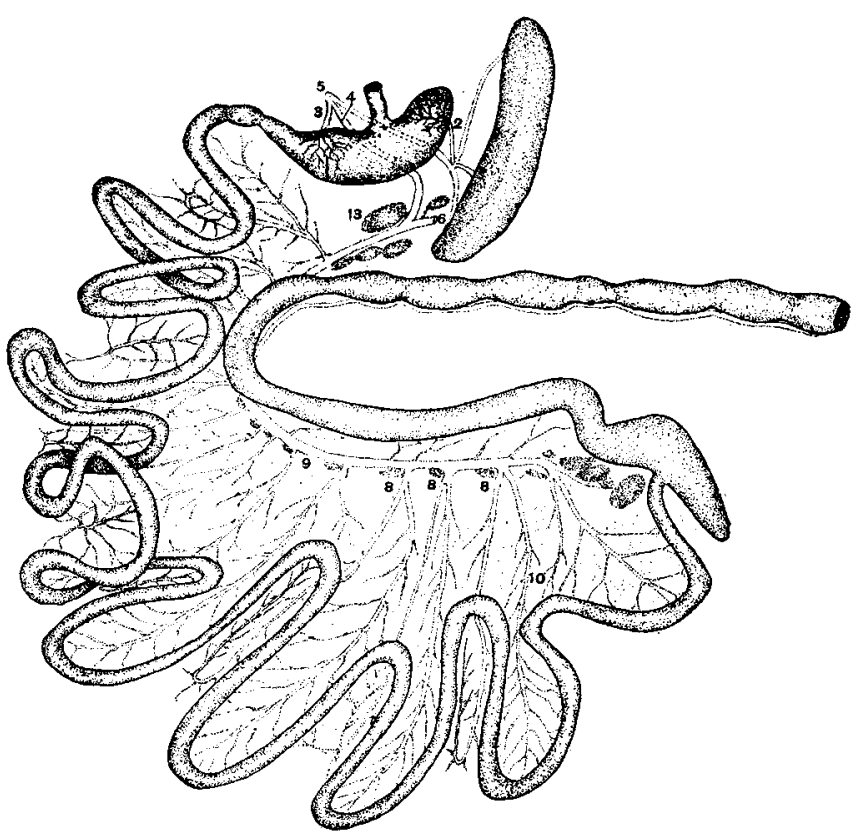

Fig. 4 The intestinal tract and lymphatics 
forward, the left lumbar node may be a considerable distance in advance of the right node. In a few instances the double nodes were divided into two single nodes, which lie some distance apart, and in two specimens an additional double node was found at the bifurcation of the ilio-lumbar vein on the right side.

The lymph vessel leading from the right lymph node (5) is so variable that a definite description cannot be given. It usually joins the left lymph vessel in some way between the lumbar node and the cisterna chyli. Figure 2, D, E, F, shows some variations of a general type. Figure $3, \mathrm{~A}$ and $\mathrm{B}$, shows two additional variations, which are more common, in the general plan, than any of the others. In only four specimens did the right lumbar lymph vessel lead to the right renal node (9) directly. In these cases a small lymph vessel tapped the renal vein from the renal node, and another vessel connected with the cisterna chyli (11), or the cisterna chyli group (12). In two specimens the right lumbar lymph vessel gave off a branch, which connected with the ilio-lumbar vein (fig. $3, \mathrm{C}$ ). This branch did not follow the veins to its tap, but went directly from the node across the ilio-psoas and psoas muscles to its juncture with the ilio-lumbar vein about $1 \mathrm{~cm}$. from the vena cava.

On the left side the. number of lumbar lymph vessels leading from the lumbar node (6) may vary from one to four, or form a network, depending somewhat on the mode of attachment with the right lymph vessel. However, all the vessels lead along the left side of the vena cava, in any case. If there is only one vessel, it will open into a single node $(10)$, just anterior to the left renal vein, from which a branch is given to the renal vein and one to the group of single nodes (12) lying to the left of the cisterna chyli. If there be more than one lymph vessel leaving the lumbar node, some one of them will enter the renal node, the rest may join the cisterna group, the cisterna directly, the renal vein directly, or any combination thereof. The latter conditions are fewer than the single vessel method.

The number of nodes in the cisterna group (12) vary from one large one to six small ones. From this group one or more 
vessels enter the cisterna chyli. Two special exceptions to this method are shown in $\mathrm{A}$ and $\mathrm{B}$ of figure 2. Drawing $\mathrm{A}$ shows both of the lumbar lymph vessels entering the cisterna chyli directly. The nodes of the cisterna group being closely collected about the periphery of the cisterna chyli, did not fill with the injection mass. The connection with the left renal vein was made from the intestinal lymph vessel (1S) through a small branch vessel $(a)$. The specimen from which drawing $\mathrm{B}$ was made, had no cisterna chyli, only a short vessel shunted off from the main thoracic duct marked its normal position (ci). The renal branch (a) came from the main lumbar lymph vessel. In both of these specimens the connection between the intestinal lymphatics and the portal vein was very prominent.

In addition to the connections with the cisterna chyli already mentioned, there is another lymph vessel received from the intestinal region past the intestinal node (13). The intestinal lymph does not pass through the intestinal node, but that the node is connected with the lymphatic system is shown by the fact that injections made from it fill the main lymph vessels. Also, the mass from the lumbar injections frequently pass into this node but not beyond it. From the cisterna chyli the thoracic duct (14) leads dorso-laterally along the superior vena cava and left innominate vein to its juncture with the venous system in the jugulo-subclavian district. Not a single specimen in the fifty rats showed the thoracic duct branching or entering the right jugulo-subclavian district, as pointed out by McClure and Silvester, ${ }^{2}$ Silvester, ${ }^{3}$ and Davis, ${ }^{4}$ in other forms.

A double lymph node is found in the groin, the inguinal nodes $(7-8)$, located in the bifurcations of the superficial epigastric vein, and so closely attached to the lower dermis that in removing the skin the node remains imbedded in the subcutaneous

$2 \mathrm{McClure}$ and Silvester. A comparative study of the lymphatico-venous communications in adult mammals. Anat. Rec., vol. 3, 1909.

${ }^{3} \mathrm{~F}$. Silvester. On the presence of permanent communications between the lymphatic and venous system at the level of the renal vein in adult South American monkeys. Am. Jour. Anat., vol. 12, 1912.

${ }^{4}$ Henry K. Davis. A statical study of the thoracic duct in man. Am. Jour. Anat., vol. 17, 1915. 
tissue. While this node is connected with the femoral lymph vessel, in only two injections did the mass run backward into it. The vessel leaving anteriorly from the inguinal node soon divides into two branches. These branches lead through the lower layers of the dermis to the axial region where they again join to enter a double node, which is usually found in the bifurcation of the lateral thoracic vein $(15-16)$. From here a lymph vessel leads to another double node $(17-18)$ located in the same general region only a little anteriorly; then to the third double node of this group (19-20) found by the intersection of the lateral thoracic and the axillary vein. This node also receives the lymph vessels from the elbow nodes (1-2). On the left side a lymph vessel leads from node 20 to the communication with the venous system, either through the thoracic duct tap (24), or through a separate tap in the immediate district. On the right side the communication is in the same relative position (23).

In the anterior part of the thorax, between the two innominate veins, are two groups of single nodes, the thoracic groups (25). Each group varies in the number of nodes it contains, from four to eight. When one node in the group is injected all the other nodes of that group fill up with the mass and show a vessel leading to the venous connection in the jugulo-subclavian district. The right group connects with the right district and the left with the left district. Injections from other parts of the lymphatic system do not show these nodes.

The tongue and lips have many small vessels which collect in larger main vessels on each side of the head. These vessels lead to the submaxillary lymph nodes (28), which lie at the branching of the external jugular vein into the anterior facial and transverse vein. From these nodes, vessels lead almost directly inward, dorsally, to single nodes lying on each side of the trachea. Further down the trachea, on each side, are two nodes lying in close proximity, one a very small single node and the other a large double node. A lymph vessel leads from the larger node to the jugulo-subclavian tap on its respective side. 
From injections in the intestinal walls a great number of small vessels are shown collecting into larger and larger vessels until they finally join the great intestinal lymph vessel, which follows the large intestine from the appendix to the intestinal lymph node in the anterior part of the abdominal cavity. In the region of the appendix there are several lymph nodes, all of which are single. In some cases they are so united as to form one large compound node, 2 or $3 \mathrm{~cm}$. long. There is usually a single node at the juncture of each mesenteric lymph vessel (8, fig. 4) with the main intestinal lymph vessel (9, fig. 4). Just a short way from the cisterna chyli is the large, single, intestinal node (13, figs. 1, 2, 4), which marks the branching of the large intestinal lymph vessel. From here one branch goes to the cisterna chyli (6) and the other to the portal vein (5). The injections made from the nodes in the region of the appendix did not always show the portal branch of the vessel, but there were a sufficient number of cases to demonstrate that such a connection is fairly common. There were a varying number of single nodes in the region of the large intestinal node which did not fill up with the mass from the intestinal injection. However, injections made from them showed that they were connected with the intestinal lymph vessels.

Injections made from the spleen (1, fig. 4) show four lymph vessels leaving at various places along the hilus, accompanied by the splenic veins. Near the cardiac end of the stomach the four splenic lymph vessels unite with a small lymph vessel from the fundus (2) to form the main splenic lymph vessel which now proceeds dorsally toward the pyloric end of the stomach, from which it receives another small lymph vessel (3). Almost immediately thereafter, the branch from the intestinal lymphatics, when present (4), and the splenic vessel unite to join the portal vein (5). 
On the nodes. There seem to be two types of nodes; (a) a single node in which the lymph enters at the periphery, passes through the body of the node to the hilus, where a lymph vessel is formed; (b) the double node, referred to above, in which two single nodes are bound together in the same capsule. Instances showing this double nature are seen when injections made dis$t^{2} l^{5}$ to a double node fill the posterior half of the node and pass on well into the vessels beyond before the anterior half of the node begins to fill; injections made in the posterior half of these nodes will not fill the anterior half for some time after the vessel leading from the node is filled; injections made in the anterior half seldom enters the posterior half; in some instances where normally a double node is found, two single nodes were found only slightly separated; and finally, dissections can usually be made, to show the double nature of the nodes. The significance of the double node has not yet been determined, but it is possible that the two parts perform separate functions. The posterior part of the node responds to the injection mass, just as the single nodes of the head, knee, elbow and caudal region do, while the anterior part responds as the intestinal and thoracic nodes. It is possible, therefore, that the posterior part and certain single nodes may act as filters primarily, while the anterior part and certain other single nodes may act as elaborators only. ${ }^{6}$ A histological study is to follow.

On the vessels. No attempt was made in this study to determine the nature of the origin of the lymph vessels in the tissues. The injections do show, however, a definite tubed system beyond the origin, carrying lymph in only one direction and that toward the venous connection. The valves in the vessels are shown by the knotted appearance of those vessels filled with the mass. In general, the lymph vessels follow the blood vessels very closely, the lumbar region being the main exception. The walls are

- The venous connection is considered the anterior end of the lymph vessel.

- Sabin, in Morris's "Human anatomy," Part II, page 702, recognizes one type of lymph node with two functions. 
very thin and easily ruptured by mechanical devices, but are capable of considerable extention in situ without injury. Where a lymph vessel passes through a node it always enters at the periphery and leaves from the hilus.

On the connections. In addition to the venous connections pointed out by McClure, ${ }^{2}$ Silvester, ${ }^{3}$ and others, there appears to be two additional connections in the rat, the portal vein connection and the ilio-lumbar connection.

The portal vein connection receives the lymph from the spleen and stomach, and part of the intestinal lymph (fig. 4, 5).

The ilio-lumbar connection receives some lymph from the right lumbar lymph vessel (fig. $3, \mathrm{C}$ ).

The left jugulo-subclavian tap, studied by McClure and Silvester in several forms, ${ }^{2}$ in the rat receives the lymph from the left side of the head and neck, the left thoracic group, the left fore limb, side and hind limb, the deep seated vessels of the right hind limb and lumbar region.

The right jugulo-subclavian tap ${ }^{2}$ receives the lymph from the right side of the head and neck, the right thoracic group, the right fore limb and the superficial vessels of the right side and hind limb (fig. 1, 23).

The right renal vein communications, studied by Silvester, ${ }^{3}$ when present in the rat, receives part of the lymph from the deep seated vessels of the right hind limb (fig. 1,9).

The left renal vein communication, ${ }^{3}$ receives part of the lymph from the hind quarters (fig. 1, 10).

The portal and ilio-lumbar vein connections have not been reported previously, to the knowledge of the writer.

On general arrangement. The lymphatic system of the trunk is sinistral in the rat. The irregular occurrence and the extremely variable connections of the right lumbar lymphatics, as contrasted with the comparatively stable left lumbar system, is evidence of this fact. In all of the appendages of the body the lymph vessels follow very closely the various branches of the venous system. When the lymphatic system is injected it is very easy to distinguish the arterial, venous and lymphatic systems, when they occur together. 
In conclusion, I take this opportunity to thank Dr. Frank A. Stromsten for his many helpful suggestions in the course of this work, and for his human interest, which has made this work possible and a pleasure. 